\title{
On the dynamical consequences of the Poynting-Robertson drag caused by solar wind
}

\author{
Galina O. Ryabova \\ Research Institute of Applied Mathematics and Mechanics of Tomsk State University, 634050 \\ Tomsk, Russia \\ email: ryabova@niipmm.tsu.ru
}

\begin{abstract}
To correctly take into account the solar wind drag equations for secular variations in the meteoroid orbital semimajor axes and eccentricities were obtained. The equations are similar to those derived for the Poynting- Robertson radiation drag (Wyatt and Whipple 1950). An estimation for the Geminid meteoroid stream shows that the ratios of corpuscular to radiation drags are $0.4-0.7$ for particles of size $>10 \mu m$, i.e. the effect is much stronger than was assumed before.
\end{abstract}

Keywords. Meteoroids, solar wind, methods: analytical, celestial mechanics

\section{Introduction}

Mukai \& Yamamoto (1982) derived an improved formula for the drag generated by motion of dust grains through solar wind. As a rule, the influence of the corpuscular analogue is taken into account by introducing the proper coefficient equal to the ratio between the corpuscular and radiation drags. The estimates of the different authors (Whipple 1967a; Whipple 1967b; Burns, Lamy \& Soter 1979; Polyakhova 1979) give close values of the order of 0.2 . The estimate of Misconi (1976) is slightly higher and equal to 0.26. Mukai \& Yamamoto (1982) found all these estimates to be underestimated. Their results were applied to study the orbital evolution of dust particles in the region of exterior mean motion resonances by Marzari \& Vanzani (1994). But they did not find application in numerous works devoted to study of mass separation in meteoroid streams (for details see Ryabova 1999) leading to overestimation of meteoroid stream's age.

Based on the results of Mukai \& Yamamoto (1982), the author derived in this paper equations for secular variations in semimajor axes $a$ and eccentricities $e$ of the particles' orbits. The influence of the corpuscular analogue of the Poynting-Robertson effect was confirmed to be stronger than it was assumed before.

Below, unless otherwise specified, we shall use CGS units.

\section{Solar wind force}

The force acting on meteoroid and caused by its interaction with the solar wind is

$$
\begin{aligned}
\mathbf{F}_{w}=f_{w} \frac{\mathbf{U}}{U} & =\left(\sum_{i=p, \alpha} \frac{m_{i} n_{i}}{2} U^{2} A C_{D, i}\right) \times \\
& \times[(W \cos \phi-\dot{r}) \hat{\mathbf{r}}+(\mp W \sin \phi-r \dot{\theta}) \hat{\boldsymbol{\theta}}] \frac{1}{U}
\end{aligned}
$$

Here $m_{i}$ and $n_{i}$ are the mass and the number density of the $i$ th component of the solar wind, respectively; $C_{D, i}$ is the drag coefficient caused by this component; indices "p" and 
" $\alpha$ " denote proton and alpha particles; $\mathbf{U}=\mathbf{W}-\mathbf{V}$, where $\mathbf{W}$ is the average velocity of the solar wind flow and $\mathbf{V}$ is the meteoroid velocity; $\hat{\mathbf{r}}$ and $\hat{\boldsymbol{\theta}}$ are the radial and transverse unit vectors, respectively; $\dot{r}$ and $r \dot{\theta}$ are the radial and transverse components of the meteoroid velocity respectively; $\theta$ is the orbital longitude; $A$ is the area of the cross-section of the meteoroid; and $\phi$ is the angle between the vectors $\overline{\mathbf{W}}$ and $\hat{\mathbf{r}}$.

Solar wind plasma consists of protons, electrons, alpha particles, and heavy ions. In equation (2.1), only protons and alpha particles are taken into account, because the contribution of electrons and heavy ions is negligible. Following Mukai \& Yamamoto (1982), we take, for the heliocentric distance $r$ (Eyni \& Steinitz 1980),

$$
n_{p}=8.1\left(\frac{r_{0}}{r}\right)^{2}\left(\frac{400 \mathrm{~km} / \mathrm{s}}{W}\right)^{2} \mathrm{~cm}^{-3},
$$

where $r_{0}=1 \mathrm{AU}$. The mean velocity of the solar wind is constant in the range $0.3<r<$ $10 \mathrm{AU}$ and equal to $400 \mathrm{~km} / \mathrm{s}$, and $n_{\alpha} / n_{p}=0.05$ (Svalgaard 1977). As a consequence $n_{p, 0} m_{p}+n_{\alpha, 0} m_{\alpha}=1.2 n_{p, 0} m_{p}=1.626 \times 10^{-23}$, where $n_{p, 0}$ and $n_{\alpha, 0}$ are the values at $r_{0}$. The average direction from the Sun of the interplanetary plasma flow is deflected from the radial. But, since the terms with $\sin \phi$ and $\cos \phi$ in (2.1) do not contribute to $<d a / d t>$, we will not discuss $\phi$. In the limit, when the dispersion in the velocity of the solar wind particles is small in comparison with the flow velocity and the sputtering process is also omitted, we have $C_{D, i} \rightarrow 2$ and $f_{w} \rightarrow f_{w 0}$. Mukai \& Yamamoto (1982) calculated $\psi=f_{w} / f_{w 0}$ in the range $0.1<r<5$ AU for the average parameters of the solar wind flow and for three grain materials : water ice, magnetite, and obsidian). The value $\psi$ proved to be nearly constant for these materials, and the following values can be used for estimations: 1.6 (water ice), 1.4 (magnetite), and 1.1 (obsidian). Taking all this into account, we replace $f_{w}$ in $(2.1)$ by its approximate expression:

$$
f_{w} \approx \psi f_{w 0}=1.626 \times 10^{-23} \psi\left(\frac{r_{0}}{r}\right)^{2} U^{2} A
$$

\section{Secular variations in semimajor axis and eccentricity}

Now we can find the time-averaged secular changes $<d a / d t>_{w}$ and $\left\langle d a / d t>_{w}\right.$ by means of the procedure used by Burns, Lamy \& Soter (1979, Sec. VII). We use the Gaussian equations for the derivatives of orbital elements in the form adopted by Burns, Lamy \& Soter (1979):

$$
\begin{aligned}
& \frac{d a}{d t}=\frac{2 a^{2}}{m H}\left[F_{r} e \sin v+F_{t}(1+e \cos v)\right], \\
& \frac{d e}{d t}=\frac{H}{m \mu}\left[F_{r} \sin v+F_{t} \frac{e+2 \cos v+e \cos ^{2} v}{1+e \cos v}\right],
\end{aligned}
$$

here $m$ is the meteoroid mass; $H=[\mu p]^{1 / 2}$ is the meteoroid angular momentum per unit mass; $p=a\left(1-e^{2}\right) ; \mu$ is the solar gravitational constant; $v$ is the true anomaly; $F_{r}$ and $F_{t}$ are the radial and transverse components of the perturbing force $\mathbf{F}_{w}$, respectively. Let us average the variation in the semimajor axis over the revolution period, assuming that $a$ and $e$ are nearly constant over this time interval and that the angular momentum is conserved. Then

$$
\left\langle\frac{d a}{d t}\right\rangle=\frac{1}{P H} \oint \frac{d a}{d t} r^{2} d v,
$$

where $P$ is the orbital period. 
Next, we replace $f_{w}$ in (2.1) by its approximate expression (2.2). Obtaining the new expression for $\mathbf{F}_{w}$, we substitute the resulting components $F_{r}$ and $F_{t}$ into (3.1), and then (3.1) into (3.2). In $F_{r}$ and $F_{t}$ only terms depending on velocity are taken into account, because the circular integral of terms independent on velocity is equal to zero. Since $\dot{r}=\mu e \sin v / H$, and $r \dot{\theta}=\mu(1+e \cos v) / H$, we have

$$
\left\langle\frac{d a}{d t}\right\rangle=\frac{1.626 \times 10^{-23} \psi r_{0}^{2}}{\pi a\left(1-e^{2}\right)^{3 / 2}} \frac{A}{m} \oint U\left[e^{2} \sin ^{2} v+(1+e \cos v)^{2}\right] d v .
$$

In the right-hand side of (3.3) we see value $U$ under the integral. This value changes along the meteoroid's orbit. It is obvious from (2.1) that

$$
\mathbf{U}=[(W \cos \phi-\dot{r}) \hat{\mathbf{r}}+(\mp W \sin \phi-r \dot{\theta}) \hat{\boldsymbol{\theta}}] .
$$

For our estimation it is quite enough to use $U$ averaged over the orbit. Let us use the same technique as above. Then

$$
\left\langle U^{2}\right\rangle=\frac{1}{P H} \int_{0}^{\pi} U^{2} r^{2} d v=\pi \mu a^{3 / 2}\left(1-2 p^{2}\right) p^{-5 / 2}-4 W a e(\mu \pi)^{1 / 2} .
$$

The equation for the eccentricity is derived in a similar way. Then after taking integrals and transformations the final equations are

$$
\begin{aligned}
& \left\langle\frac{d a}{d t}\right\rangle_{w}=-3.65 \times 10^{3} \psi \hat{U} \frac{A}{m} \frac{2+2 e^{2}}{a\left(1-e^{2}\right)^{3 / 2}}, \\
& \left\langle\frac{d e}{d t}\right\rangle_{w}=-3.65 \times 10^{3} \psi \hat{U} \frac{A}{m} \frac{2 e}{a^{2}\left(1-e^{2}\right)^{1 / 2}} .
\end{aligned}
$$

The values are obviously comparable with those, derived for the Poynting-Robertson effect, and should be taken into account in estimating the stream age.

\section{Estimation for the Geminid meteoroid stream}

For the orbit of asteroid Phaethon, the parent body of the Geminids (with $a=$ $1.323 \mathrm{AU}$ and $e=0.891$ ), and a solar wind velocity $W$ (directed radially for simplicity) of $400 \mathrm{~km} / \mathrm{s}, U$ varies from 355 to $450 \mathrm{~km} / \mathrm{s}$, the averaged value over the orbit $\hat{U}$, calculated from (3.4), being $386 \mathrm{~km} / \mathrm{s}$. Because the uncertainties in $n_{p}$ and $W$ are much larger, we take $U / W \approx 1$ for the Geminids.

The ratios between corpuscular and radiation drags for water ice, magnetite, and obsidian are equal to about $0.55,0.48$, and 0.38 respectively, for the coefficient of Mie scattering $Q_{p r}=1$. If we take into account, for example, that $Q_{p r} \approx 0.53$ for obsidian grains of 10 to $100 \mu \mathrm{m}$ in size (Mukai \& Yamamoto 1982), then the above ratio will increase to 0.71 .

What consequences these results have for the Geminid stream evolution study? Estimations of the Geminid's age on secular variations in $a$ and $e$ due to the Poynting-Robertson effect only are in the range from 4700 to 6700 years (a review can be found in Ryabova 1999) for spherical particles. Correction for the solar wind decreases the estimated age to $3.1-4.8$ thousand of years, and correction for nonspherical shape (Ryabova 1999) gives the final value $2.1-3.2$ thousand of years.

\section{Acknowledgements}

The author thanks IAU and the organizers of the Colloquium for their support. 


\section{References}

Burns, J.A., Lamy, Ph.L. \& Soter, S. 1979, Icarus 40, 1

Eyni, M. \& Steinitz, R. 1980, in: M.Dryer \& E.Tandberg-Hanssen (eds.), Solar and Interplanetary Dynamics, Proc. IAU Symp. No. 91, p. 147

Marzari, F. \& Vanzani, V. 1994, Astron. Astrophys. 283, 275

Misconi, N.Y. 1976, Astron. Astrophys. 51, 357

Mukai, T. \& Yamamoto, T. 1982, Astron. Astrophys. 107, 97

Polyakhova, E.H. 1979, Uchenye zapiski LGU 400, 161 (in Russian)

Ryabova, G.O. 1999, Solar System Research 33, 258

Svalgaard, L. 1977, in: A.Bruzek \& C.J. Durrant (eds.), Illustrated glossary for solar and solarterrestrial physics (Astrophys. and Space Sci. Lib. Vol.69), p. 149

Whipple, F.L. 1967a, Smithsonian Astrophys. Obs. Spec. Rept. 239, 1

Whipple, F.L. 1967b, in: J. Weinberg (ed.), The Zodiacal Light and the Interplanetary Medium (NASA SP-150), p. 409

Wyatt, S.P. \& Whipple, F.L. 1950, Astrophys. J. 111, 134 